\title{
Pericia, razonamiento probatorio y diseño institucional
}

\author{
Expert Evidence, Evidential Reasoning and \\ Institutional Design
}

Valeria Trotti*

Recepción y evaluación de propuesta: 06/05/2018

Aceptación: 10/06/2018

Recepción y aceptación final: 16/06/2020

Resumen: Este artículo consiste en la presentación del debate entablado entre Carmen Vázquez, Florencia Rimoldi y Rachel Herdy acerca de ciertos modelos de justificación de la prueba pericial. Sobre las bases que estos modelos arrojen, es posible evaluar las herramientas procesales a legislar y el tipo de práctica que cabe emprender respecto de este tipo de prueba en el contexto del proceso judicial. Al final, plantearé algunas reflexiones pendientes sobre esos puntos.

Palabras clave: Justificación de la prueba pericial, diseños institucionales, contexto procesal.

\begin{abstract}
This paper consists in an introduction to the debate between Carmen Vázquez, Florencia Rimoldi and Rachel Herdy about different models for the justification of expert evidence. Based on these models' grounds, it is possible to assess which procedural tools should be enacted by the legislator and the type of practice that must be implemented regarding this type of

Magíster en Derecho y Argumentación, Universidad Nacional de Córdoba, Argentina y Magíster en Razonamiento Probatorio, Universidades de Girona, España y Génova, Italia. Correo electrónico: valetrotti@hotmail.com.
\end{abstract}


evidence, in the context of the judicial process. By way of a conclusion, I will point out some pending reflections regarding these points.

Keywords: Expert evidence justification, institutional design, procedural context.

\section{Introducción}

Bajo un paradigma de racionalidad, ${ }^{1}$ una heterogénea comunidad de juristas, filósofos/as, epistemólogos/as y sociólogos/as han elaborado numerosos trabajos sobre los hechos y la prueba en contextos jurídicos. La transversalidad de sus enfoques referidos a estas áreas del conocimiento jurídico ha puesto en debate muchos presupuestos teóricos y normativos que inciden en los sistemas jurídicos y sus prácticas. Actualmente, estos debates presentan nuevos bríos y se orientan a renovar diseños institucionales.

Este número de Discusiones es una muestra a menor escala del trabajo de esa comunidad. A partir de un modelo de comprensión de los elementos que componen el razonamiento pericial, Carmen Vázquez propone un diseño institucional de la prueba pericial que supone la disposición en conjunto de herramientas procesales y la fijación de pautas para la gestión de su práctica. A su criterio, un diseño como este permitirá a los tribunales $\mathrm{y}$ a las partes lidiar de mejor manera con el conocimiento experto que producen peritos/as en el proceso. A continuación, Florencia Rimoldi y Rachel Herdy analizan su texto ancladas en modelos de justificación epistémica y normativa, parcial o totalmente, diferente al propuesto por Vázquez. Luego de un contradictorio en diferido, esta última puntualiza y amplía ciertos aspectos de su intervención inicial, especialmente, los referidos a los presupuestos que apoyan su modelo.

Un repaso de textos anteriores de esta autora $-\mathrm{y}$ otros que rodean la discusión- muestra al menos tres puntos básicos que operan explícita o implícitamente en este intercambio de opiniones en particular. Uno, la

1 Aunque todavía impreciso, este paradigma agrupa a una serie de estudios sobre la prueba y los hechos en contextos procesales (Accatino, 2019); una recopilación de las teorías epistemológicas que lo integran, pero a partir de diferentes criterios metodológicos (Marrero, 2015). 
importancia de precisar teóricamente el concepto de testimonio experto que subyace en la prueba pericial. Dos, la directiva de institucionalizar ese concepto a partir de las bases epistémicas adecuadas que guían el testimonio experto. Tres, la observación que metodológicamente el debate normativo se asienta en los dos primeros.

En lo sustancial, presentaré al lector o lectora un mapa de la discusión que encontrará sobre estas cuestiones. ${ }^{2} \mathrm{Al}$ final, plantearé algunas deudas que quedan pendientes de reflexión para el futuro. En cuanto al mapa, lo que diga al respecto -e incluso más allá de mis propios errores en su formulación - siempre será limitado. Una adecuada comprensión de las ideas de las autoras exige continuar con la más estimulante lectura de las páginas que siguen.

\section{Breves notas sobre la justificación racional de la prueba de los hechos en la decisión judicial}

En parte, el debate sostenido en esta ocasión pone en cuestión los modelos que deben guiar la justificación racional de la inferencia probatoria contenida en la pericia y que integra el razonamiento probatorio en que se apoya la decisión que resuelve un caso judicial. Una muy breve reseña de las estructuras teóricas y normativas que subyacen a la valoración racional de esa inferencia y la juzgan, ${ }^{3}$ por un lado, pone en contexto este objeto de

2 Resulta una casualidad inusual que estos trabajos salgan a la luz mientras transitamos una pandemia producida por el contagio masivo del virus denominado SARS-CoV-2 que causa la enfermedad infecciosa COVID-19. Esta pandemia nos ha exigido en diversos ámbitos institucionales, sociales y personales tomar decisiones apoyadas, especialmente, en los testimonios de expertos/as. Si bien, como veremos, las autoras han delimitado el debate de la valoración del testimonio experto al contexto judicial, muchos de sus argumentos y conclusiones podrían pensarse respecto de otros contextos decisionales.

3 Más allá de los heterogéneos debates que se libran en orden a las cuestiones que involucra afirmar que un hecho ha sido probado, existe cierto acuerdo terminológico y conceptual que resulta útil para esta presentación. Textos elementales sobre estos debates y acuerdos son, entre otros, Gascón Abellán, 2004; Ferrer, 2005, 2007, 2018; González Lagier, 2018; Bayón, 2009; Dei Vecchi, 2020. 
análisis. Pero, por otro, permitirá luego identificar ciertas líneas de trabajo que pueden complementar estos estudios u orientar otros en el futuro.

En este orden, y en cualquier caso, la justificación referida a la prueba de los hechos debe revisarse bajo la idea de que esa tarea coloca a quienes la juzgan en un contexto de incertidumbre insuprimible y, por ello, sus enunciados son falibles indefectiblemente. Sin embargo, esa justificación adquiere diferentes matices según qué tipo de decisión esté en juego, quién la formule y en qué tiempo. Esto particularmente porque, en el marco de un proceso judicial, las probabilidades de acierto de la decisión judicial sobre los hechos del caso se reducen dadas ciertas reglas sobre la prueba ${ }^{4}$ y vista la limitación temporal que impone la resolución institucionalizada del conflicto en un lapso determinado.

Ahora bien, la justificación del enunciado que refiere un determinado hecho $(p)$ como parte de la premisa fáctica del silogismo lógico de la decisión judicial final resulta de la aceptación o no de que "p está probado". "Está probado que $p$ ” es un enunciado cuya corrección resulta, a su vez, del razonamiento probatorio en el que se apoya. Esa corrección del razonamiento probatorio, finalmente, depende del tipo de razones que lo integran.

Bajo los sistemas de valoración racional o sana crítica racional, ${ }^{5}$ estas razones son de dos tipos: explicativas o epistémicas y morales o políticas. En un sentido muy básico, las razones morales o políticas determinan si

4 Estas reglas dependen del sentido que se asigne a la expresión prueba. Una distinción ya clásica es entender a la prueba como medio, actividad o resultado (Gascón Abellán, 2004, pp. 84-85; Ferrer Beltrán, 2005, pp. 39-43). Ferrer Beltrán identifica tres tipos de reglas sobre la prueba de acuerdo a esos diferentes significados; según esa identificación, analiza en qué medida esas reglas pueden favorecer o no a la averiguación de la verdad que actúa como uno de los objetivos institucionales del proceso (Ferrer Beltrán, 2005, pp. 40-48, y 2007, pp. 35/36).

5 La idea de valoración judicial de la prueba tiene cabida cuando el razonamiento probatorio se estructura mediante una inferencia probatoria empírica según la cual los enunciados de los hechos a probar (o hipótesis) y los elementos de juicio o datos probatorios se correlacionan mediante una conexión o enlace empírico. Otros tipos de enlaces que definen otros tipos de inferencias pueden ser normativos (i. e. las presunciones de fuente legal o jurisprudencial) y conceptuales (i.e. aquellos que dependen de una definición o relación conceptual), ver González Lagier (2018). Ambos se dejan de lado aquí pues, en definitiva, los elementos de juicio que arrojan las pruebas periciales resultan premisas de las inferencias probatorias empíricas. 
las pruebas valoradas (mediante razones epistémicas) en un caso judicial son suficientes para dar por probada una hipótesis y si la distribución de riesgos de error (falsos positivos o negativos) es adecuada. El juicio sobre cuya base se define esa suficiencia probatoria es lo que ampliamente se conoce como estándar de prueba.

Las razones explicativas o epistémicas resultan del uso de criterios propios de la epistemología jurídica. A través de estos criterios es posible evaluar la solidez de los indicios o elementos de juicio según los cuales se avalan o corroboran los hechos a probar o hipótesis afirmadas en el enunciado probatorio.

Más en detalle, la solidez de las premisas de este tipo de inferencia probatoria depende de la revisión de cada uno de sus elementos (i.e., datos probatorios, generalizaciones y hechos a probar). En la inferencia probatoria epistémica, los elementos de juicios se conectan con el hecho a probar mediante un enlace empírico, esto es, mediante un enlace que se identifica con reglas o máximas de experiencias o, más técnicamente, generalizaciones basadas en el conocimiento científico o en el sentido común o la experiencia. Finalmente, ese enlace encuentra apoyo en un determinado respaldo o fundamento (González Lagier, 2018).

La identificación y relevancia teórica y normativa de estas distinciones, además, debe evaluarse según se siga una estructura del razonamiento probatorio orientada a partir de concepciones normativas holistas o atomistas del objeto de prueba y de las razones de prueba. ${ }^{6}$ Grosso modo, en cualquier caso, el modelo holista atiende especialmente a criterios de coherencia en la definición de la mejor hipótesis probada; en cambio, el modelo atomista, en versiones modernas del chart method presentado por Wigmore, categoriza y distingue analíticamente los hechos del caso a probar como probandum final (i.e., premisa menor del silogismo judicial), penúltima o intermedia,

6 En su reconstrucción de la discusión holismo/atomismo como modelos que inciden en el razonamiento probatorio, Accatino individualiza diferentes sentidos sobre los cuales es posible afirmar, según cada modelo, que se tiene "una concepción (o un conjunto de tesis acerca) del razonamiento probatorio". Con esas ideas, entiende que la definición de una concepción normativa de la justificación probatoria exige distinguir entre los momentos del razonamiento probatorio, sus perspectivas (i. e. descriptivas o normativas) y si a través de él se trata del objeto de la prueba (probandum) o de razones probatorias (probans) (Accatino, 2014). 
a fin de revisar la fuerza de las inferencias probatorias que integran gráficamente la cadena de razonamientos probatorios, pues todas ellas son fuentes potenciales de incertidumbres (Anderson, Schum y Twining, 2015, p. 96).

Por su parte, es relevante distinguir ciertas relaciones entre los sujetos y momentos procesales que operan en el contexto procesal y condicionan la justificación del enunciado probatorio tal como fue expuesto. Estas relaciones, a diferencia de lo anterior, transitan más explícitamente los artículos de Vázquez y los comentarios expuestos por Rimoldi y Herdy.

En efecto, las inferencias probatorias que integran el razonamiento probatorio pueden ser elaboradas por quienes producen la prueba (en la literatura procesal, los órganos de prueba), quienes alegan sobre ellas en razón de sus pretensiones en el juicio (las partes) o quienes deciden finalmente esos casos (los tribunales integrados por jurados o no). Además, estas inferencias se constituyen en el proceso a través de diferentes pruebas que son evaluadas según los momentos procesales que atraviesan (i. e., admisión, práctica y valoración).

Finalmente, el razonamiento probatorio puede integrarse por más de una inferencia probatoria según sea que ellas se refieran a la prueba no del enunciado probatorio ("está probado que p") sino de los elementos de juicio (o indicios) que configuran premisas de ese razonamiento, como se indicó antes. Cuando estas inferencias probatorias son configuradas a partir de las diferentes variantes de personas procesales y momentos en el marco de la prueba pericial, se la denominará como inferencia probatoria pericial. La combinación de esos elementos lleva a que la inferencia probatoria pericial pueda ser formulada por los/as expertos/as, las partes o los tribunales durante la admisión, práctica y valoración.

Esto pone en escena que constituye un problema sumamente complejo conceptualizar y justificar las acciones tomadas por quienes participan en el proceso, especialmente el juez o jueza, vinculadas con el tratamiento de la información probatoria - y otra que la complementa - contenida en esa inferencia y que conducen y definen respectivamente estos sujetos del proceso en sus diversos tiempos. Pero no debe perderse de vista que tales propósitos no deberían ir muy lejos de los presupuestos teóricos y normativos que rigen la justificación del enunciado probatorio. 


\section{Dificultades y errores en el diseño institucional de la prueba pericial}

Ambos artículos de Carmen Vázquez, el inicial y el final, ofrecen una propuesta de trabajo que intenta dar cuenta de las complejidades resaltadas.

En ese sentido, afirma que una decisión judicial - que se apoye en este tipo de prueba - será racional si hubo una adecuada comprensión de las inferencias probatorias periciales. Esa comprensión debe alcanzar prioritariamente los elementos que la conforman, en sus palabras, las generalizaciones expertas, la identificación de los datos relevantes del caso y las relaciones entre estos enunciados. El resultado de esta comprensión resulta una condición necesaria para un adecuado control de la fiabilidad de la evidencia aportada a través de la pericia. De tal estado de cosas se sigue una exigencia suplementaria de lege ferenda - o, al menos, de sententia ferenda- de identificar, diseñar o rediseñar sistemas normativos que integren herramientas procesales cuyo objeto sea optimizar ese control. De igual modo, considera relevante el manejo y disposición de la información que peritos/as, partes y tribunales posean y sobre la cual versará la pericia.

Su interés por examinar estas cuestiones resulta especialmente de la deficiente regulación que los sistemas jurídicos —en particular, el continental - han dado a la admisión, pero especialmente a la práctica y a la valoración de la prueba pericial. Una muestra de esto la observa en numerosos incentivos procesales que disimulan en todos estos tramos una inadecuada deferencia a los argumentos y conclusiones de los/las peritos/as. Estos incentivos son producto de sistemas jurídicos que prevén medidas de control que no hacen foco en la fiabilidad de la inferencia probatoria pericial sino en la justificación que se encuentra fuera de ella. ${ }^{7}$

Estos defectos de los sistemas jurídicos y sus prácticas —esto es, deferencia e incentivos a la deferencia- llevan a Vázquez a presentar una propuesta normativa para resolver estas cuestiones. La revisión de este estado de cosas,

7 En su descripción crítica de estos incentivos, Vázquez identifica que los/las jueces/zas cuentan con, o se hacen de, herramientas para admitir, practicar y valorar esta prueba a partir de condiciones referidas a quien realiza la pericia, a pautas formales de los informes periciales (como son la completitud o no contradicción) o al carácter científico o no del objeto pericial, entre otras. 
en cambio, puede variar si se siguen modelos teóricos y normativos alternativos a esta prueba como los que oponen Florencia Rimoldi y Rachel Herdy.

Ambas autoras no objetan que sea un punto de interés de los sistemas jurídicos actuales perfilar nuevos diseños institucionales de la prueba pericial o, con más consenso, reforzar los ya existentes. Con acuerdos y desacuerdos de distinto tipo y alcance, reexaminan los "defectos" que identifica Vázquez como tales a partir del contraste que existe entre los presupuestos teórico-epistémicos que ella asume inicialmente y los que consideran adecuados sus comentaristas. Una peculiaridad, que se observa con matices, resulta de la falta de discusión por parte de las comentaristas de las propuestas de Vázquez para incorporar en conjunto a las legislaciones herramientas procesales y acerca de las gestiones que le caben a la práctica de la prueba pericial.

Esa ausencia debería revelar o que los desacuerdos no son genuinos lo que es difícil de demostrar-, o que existen diferencias sobre esos puntos simplemente omitidas o no desarrolladas suficientemente por límites de espacio y tiempo.

\section{Sobre los modelos de justificación de la inferencia probatoria pericial}

Las autoras acuerdan en que es diferente que el juez o jueza posea información pericial a que la comprenda. Desacuerdan, en cambio, sobre qué deben comprender, cómo o cuánto. ${ }^{8}$ Inicialmente, Carmen Vázquez apoya su propuesta normativa en el rechazo de los presupuestos teóricos seguidos por el paternalismo epistémico de Goldman (1991) respecto de la capacidad cognitiva de quienes juzgan y deciden los casos (jueces/zas o jurados/ as). Dicho apoyo también se sostiene en elementos propios de la episte-

8 Aunque no es del todo claro de qué modo - conceptual o normativamente- inciden esos desacuerdos en los elementos de la inferencia probatoria pericial; ello, particularmente, vistas las configuraciones que modelos atomistas u holistas de la justificación de la decisión judicial traen a colación en orden a los datos probatorios y razones probatorias. 
mología del testimonio dado que así se trasmite el conocimiento experto en el contexto judicial.

La autora entiende que si, como el paternalismo epistémico afirma, quienes deciden muestran defectos cognitivos sistemáticos que les impiden evaluar la calidad de la inferencia probatoria pericial, entonces corresponde revisar la decisión política de mantenerlos en esa función. Así, resulta un sinsentido desconfiar de su comprensión, pero confiar de su decisión.

Según Vázquez, no es claro que estos defectos cognitivos se sustenten en estudios empíricos que justifiquen su consideración. ${ }^{9}$ En cambio, el estado actual de los sistemas de tradición continental revela que la práctica judicial alrededor de la prueba pericial - por disposición, por contexto o por inadecuada formación universitaria - se asienta en errores e incentivos que llevan a quienes deciden a desvincularse de una mejor valoración de su contenido. A la par de ello, tampoco los sistemas jurídicos actuales -o criterios jurisprudenciales- prevén soluciones para las complejidades propias de este tipo de prueba.

Lo dicho evidencia la necesidad de educar, especialmente, a los/as jueces/zas para un mayor y mejor acercamiento al conocimiento experto y diseñar o modificar los sistemas jurídicos mediante ciertas herramientas procesales que favorezcan su comprensión. Dirigir su propuesta a la formación de jueces/zas, según dice luego, es más sencillo que hacerlo respecto de jurados/as que intervienen esporádicamente en la administración de justicia, a la par que ya existen recursos destinados a esos efectos para los/ as primeros/as. ${ }^{10}$

En adición, Vázquez refiere que el conocimiento experto se trasmite mediante el testimonio del experto en el contexto judicial. Allí, el/la perito/a y el/la juez/a son agentes epistémicos con diferentes roles, el primero

9 En cierto sentido, de hecho, en su trabajo final desalienta el uso de estudios empíricos para revisar esta cuestión o, al menos, entiende que su uso debe ser muy cuidadoso teniendo en miras el contexto en el que se producen y sus objetivos. Y más directamente sostiene que no observa en las réplicas a su trabajo algún estudio que dé cuenta de la (in)capacidad de los jueces para comprender el razonamiento pericial.

${ }^{10}$ Vale apuntar que la decisión política de quien debe juzgar los hechos no solo depende de una cuestión epistémica, sino también valorativa. Y en ello, el desplazamiento de los jurados puede resultar una dificultad suplementaria. 
actúa como hablante que transmite información, el segundo como su audiencia. Ese contexto facilita la incorporación de elementos para delimitar, hacer circular y comprender la información que es relevante. Esto, otra vez, es importante pues el juez o jueza debe ofrecer sus propias razones para coincidir, o no, con las afirmaciones formuladas en el razonamiento inferencial contenido en la pericia.

En sus réplicas, añadirá que el contexto judicial en que todo esto ocurre, y que debe considerarse para analizar ese intercambio de información, es más amplio pues también concurre la acción cooperativa - no como comunidad - de las partes y las comunidades expertas. Ello, además, debe analizarse bajo la idea de división del trabajo cognitivo de quienes intervienen en el proceso como agentes cognitivos.

Florencia Rimoldi y Rachel Herdy revisaron estas ideas y delimitaron aspectos relevantes que consideran discutibles. En el marco de esta comunidad epistémica dispuesta a baja escala, sus comentarios resultan atractivos porque han sido elaborados desde perspectivas y criterios metodológicos diferentes, a pesar de lo cual encuentran puntos de contacto. Pero también su diverso tránsito, inicial o más avanzado, renueva y profundiza el debate en la discusión acerca de los modelos de justificación de la prueba pericial enmarcados en los paradigmas de la educación o la deferencia.

Las tres rechazan los extremos que pueden tener lugar dado el tipo de relaciones que existen en el intercambio epistémico entre jueces/zas y peritos/as. Así, niegan que sean pares epistémicos pues resulta un sinsentido pretender jueces o juezas especialistas en todas las disciplinas propias de las ciencias forenses o que puedan adquirir relevancia en un caso judicial. También rechazan que la diferencia epistémica entre ellos sea tal que los primeros están completamente inhabilitados para formular cualquier clase de crítica a los argumentos y conclusiones de los/as peritos/as. ${ }^{11} \mathrm{Sin}$

${ }^{11}$ Aunque serían excepcionales, podrían presentarse casos en los que la sofisticación de cierta teoría involucrada en la inferencia probatoria pericial (como algunas de la física teórica, por ejemplo) impida cualquier revisión crítica de los/as juzgadores/as. En su primer texto, Vázquez afirma que este tipo de conocimiento, dada su imposibilidad de revisión, debería ser inadmitido en el proceso. Para un ejemplo del tipo de conocimiento que podría estar excluido, vale recurrir al ensayo sobre divulgación de Ernesto Sábato en Uno y el universo (1945). Allí, el autor se imagina intentando explicar la teoría de la relatividad a un amigo 
embargo, este consenso básico se desarma al momento de fijar el tipo y grado de comprensión, control o deferencia que cabe asumir en la revisión de diseños normativos de esa interacción, sean actuales o futuros.

Apoyada en ideas propias de la epistemología de la comprensión, Rimoldi incorpora distinciones teóricas a los conceptos de comprensión y testimonio (experto) analizados por Vázquez. Estas distinciones le permiten luego ensamblar su modelo de argumentación deferencial crítica como primera cuestión epistemológica relevante, que a su criterio mejora el contexto epistémico en el que se sitúa la interacción juez/a-perito/a.

Para esta autora, comprender un fenómeno significa captar ciertas relaciones de sus partes entre sí y con el contexto — relevante- con el que están conectadas. En ese orden de cosas, comprender el razonamiento inferencial pericial requiere que el tribunal, a través de reflexiones contrafácticas, sea capaz revisar los márgenes de error o de probabilidad de cada evidencia en juego y cómo impactaría, en su caso, un mayor margen de error o una menor probabilidad en la decisión final, vistas las opciones de decisión que deba tomar. Finalmente, como la comprensión admite grados, el juez debe buscar una que sea suficiente para valorar adecuadamente la prueba, pero no equivalente a la del experto pues directamente lo sustituiría en su práctica. $^{12}$

Rimoldi traza ese límite con aportes de la epistemología del testimonio que la diferencian, parcialmente, de la propuesta de Vázquez. Su modelo presupone que la aceptación del testimonio experto, como fuente de conocimiento o creencia justificada del juez o jueza, es el resultado de un intercambio epistémico complejo, pues en parte es argumentativo y en parte testimonial del perito al tribunal. Esta dinámica, según afirma, constituye el contexto que enmarca la argumentación deferencial crítica a la que deberían someterse jueces/zas y peritos/as y que, además, permite

mostrando cómo la primera explicación es incomprensible, la segunda es más accesible sin ser exitoso su entendimiento y la última logra finalmente su comprensión, pero de algo muy diferente de lo que la teoría es.

12 Otras razones adicionales justifican esta limitación. Así, la exigencia de imparcialidad cognitiva de los peritos testeada a través del contradictorio (Vázquez, 2018, pp. 81-90) se vería afectada si el juez evaluara los elementos de la inferencia según sus propios conocimientos. 
llevar adelante la meta de obtener una comprensión suficiente para valorar la prueba adecuadamente. ${ }^{13}$

Como segunda cuestión epistemológica relevante, Rimoldi asume que una justificación epistémica correcta del razonamiento inferencial pericial debería seguir ciertos postulados contextualistas. Y por ello desarrolla una forma especial de contextualismo que entiende adecuado a esos fines y según la cual cabe identificar cuatro factores importantes (i.e., semánticos, metodológicos o disciplinares, dialécticos y económicos o pragmáticos) que inciden en los intercambios epistémicos entre jueces/zas y peritos/as. Entre esos factores, el dialéctico y el económico o pragmático cobran una relevancia especial para configurar el contexto epistémico que condiciona ese intercambio.

Al comprometerse con estas distinciones teórico-epistemológicas, Rimoldi se diferencia de Vázquez en el grado y tipo de comprensión que cabe exigir a los jueces en el intercambio epistémico que practican con el perito. ${ }^{14} \mathrm{~A}$ lo que adiciona la relevancia de definir los factores que condicionan el contexto en el cual estos agentes epistémicos interactúan.

De modo diferente, Rachel Herdy reconstruye las tesis de Vázquez y ciertos presupuestos que asume se encuentran vigentes en ellas. Frente a ello, presenta sus mayores diferencias con esa propuesta en orden al énfasis que asume se coloca — basado en estudios empíricos- a la capacidad cognitiva de quienes juzgan (tribunales legos o no) y su habilidad para adquirir conocimiento experto; de igual modo, y conectado con este rechazo, niega que estos juzgadores deban ofrecer razones - teórico-epistémicas- de primer orden como las que elaboran los expertos.

Ambas autoras han transitado y son referentes dentro del amplio debate que existe acerca de la justificación de la prueba pericial, enmarcado en los paradigmas de la educación o la deferencia. Es así que Herdy entra a la discu-

${ }^{13}$ Justifica que esta idea resulta asequible vista la comparación con lo que ocurre con la lectura de un artículo académico orientado a personas ajenas al campo de investigación que se trata. En algún momento, el lector defiere a referencias teóricas que no es razonable que constate.

${ }^{14}$ Sobre esto, luego Vázquez advertirá que la deferencia que invoca remite más bien a la idea de acordar con otro. Esta réplica parece mostrar la necesidad de formular mayores precisiones a varios de los conceptos involucrados. 
sión detallando sintéticamente el contexto de descubrimiento de este debate al mostrar el origen de esos paradigmas -dado principalmente por el trabajo inicial de Miller y Allen (1994) y la jurisprudencia de la Corte norteamericana (conocida como "la trilogía Daubert")— ${ }^{15}$ para luego reconducirla hacia los principales argumentos que sendos paradigmas siguen.

$\mathrm{Al}$ contextualizar ese debate, esta autora señala que ciertas herramientas procesales como las que promueve Carmen Vázquez pueden ser utilizadas en ambos modelos. Pero advierte que muchas de las tesis del modelo de la educación, entre las que sitúa la propuesta de Vázquez, se sostienen según erradas bases empírico-psicológicas y teórico-epistémicas.

Sobre las bases empírico-psicológicas, refiere que Vázquez asume que quienes juzgan el testimonio experto, empíricamente, poseen capacidades para evaluar autónomamente el razonamiento probatorio pericial involucrado o pueden adquirir habilidades a esos efectos. Contextualiza este cuestionamiento en razón de estudios empíricos que muestran que jurados/ as, normalmente, para evaluar el testimonio experto siguen una estrategia periférica - esto es, aquella que recurre a indicadores para confiar en el experto o en la información que provee-, y con mucha menos frecuencia recurren a una estrategia central - es decir, aquella que exige revisar cuidadosamente esa información-.

Tal estado de cosas, adelanta Herdy, no cierra la discusión pues podría ser irrelevante cómo jurados/as evalúan esta prueba, sino cómo jueces/zas lo hacen o, más precisamente, cómo podrían hacerlo si adquieren habilidades a esos efectos. A pesar de que no hay investigación empírica suficiente respecto de la valoración de jueces/zas del testimonio experto, argumenta que igualmente es posible asumir que lo hacen de modo análogo a los/ as jurados/as. Esta analogía se funda en que la valoración del testimonio experto de jurados/as no es de menor calidad epistémica que la que hacen los/as jueces/as y, en ese sentido, jueces/zas no lo harían de modo tan diferente. ${ }^{16}$ Dada esta analogía en cuanto a las capacidades cognitivas

15 Sobre estos casos resueltos por la Corte Suprema de Estados Unidos, véase Vázquez, 2015, pp. 96 y ss.

16 Existen trabajos que - más allá de mostrar sus propias dificultades descriptivas y normativas- presentan interesantes comparaciones de cómo actúan jueces/zas y jurados/as en la toma de decisiones (Bornstein y Greene, 2017, cap. XIII; Seidman y Rose, 2005). 
relevantes, al igual que jurados/as, jueces/zas no escogen habitualmente la estrategia central para evaluar el testimonio experto, sino la periférica.

Esta autora añade que la adquisición de habilidades para evaluar de modo central el testimonio experto exige una cargosa inversión de recursos con inciertos resultados en la administración de justicia. Ella es una de las razones que desalientan propuestas de este tipo. Y lo es, además, porque entiende que las bases teórico-epistémicas que demandan esa inversión parten de una idea inadecuada de racionalidad.

Al contrario de lo que sostienen los adherentes a un modelo educacional, Herdy estima que diferir la autoridad epistémica en el perito/a no resulta un recurso irracional que deslegitime la decisión judicial. Su modelo deferencial crítico presupone que la ausencia de paridad epistémica entre jueces/zas y peritos/as impide que quienes deciden el caso judicial puedan efectuar una adecuada consideración de razones de primer orden. Ello la lleva a sostener que las razones de las que se valen los tribunales en la evaluación de la inferencia probatoria son de segundo orden, que se identifican con indicadores epistémicos (periféricos). A su criterio, este tipo de racionalidades se sostiene en la existencia de criterios precisos de control de esos indicadores, configura un modo habitual de racionalidad práctica en el contexto judicial ${ }^{17}$ y posee una mayor legitimidad democrática.

\section{Diseño institucional de la prueba pericial}

Carmen Vázquez destaca que uno de los objetivos centrales de su texto inicial lo configura la identificación e incorporación de herramientas procesales a las legislaciones para proyectar un diseño institucional de la prueba pericial que contemple de mejor modo sus complejidades. El uso de estas

17 Puntualmente sobre esto, Carmen Vázquez reflexiona que este tratamiento del argumento de la autoridad presenta dos errores que surgen de "poner el énfasis en el argumento de autoridad y obviar las falacias de autoridad..." y de "no distinguir la autoridad teórica (sobre qué creer) de la autoridad práctica (sobre qué hacer)". A continuación, expone en qué medida el argumento de la autoridad epistémica en el caso de expertos/as resulta relevante para evaluar la autonomía del razonamiento de quien se apoya en ella. 
Pericia, razonamiento probatorio y diseño institucional

herramientas reducirá ciertas dificultades que, fundamentalmente, se presentan durante su práctica o valoración.

Entre esas herramientas, enuncia la posibilidad de efectuar preguntas aclaratorias durante el contradictorio, recurrir a asesores expertos para casos complejos, realizar meta-pericias para complementar las pruebas periciales ya practicadas y careos entre expertos para afrontar los desacuerdos entre los mismos. También añade otros modos de gestionar esos desacuerdos entre peritos mediante hot-tubbing o junta de peritos, e incluso admite para casos muy complejos, aunque excepcionalmente, la integración del tribunal con un perito.

Estas herramientas, además, deben ser implementadas considerando que el proceso se integra con diferentes agentes cognitivos - juez/a, perito/a, partes y comunidades expertas - entre los cuales se divide el trabajo cognitivo de modo cooperativo, debiéndose revisar cómo optimizar esos esfuerzos de modos compatibles con sus intereses y el objetivo final de lograr una decisión judicial racional.

En adición, la autora advierte que se ha subestimado indebidamente la práctica de esta prueba, la cual es central para sentar las bases de la valoración posterior del juez o jueza. Un diseño de esta prueba como el que presenta encontrará mejor cauce si se disponen ciertas condiciones para su uso. ${ }^{18}$

Esta es - reducidamente - su propuesta institucional. Como sugiere Vázquez en sus réplicas, la revisión de la práctica de la prueba pericial y las herramientas que permiten mejorar su comprensión resultan un aporte significativo visto cierto vacío que la literatura procesal ha dejado a ese respecto.

Al margen de cuánto han reflexionado Rimoldi y Herdy sobre ello, ambas han hecho - o es posible inferir que pueden hacer- algunas consideraciones. Estas autoras oponen —o pueden oponer-ciertos reparos a la admisión

18 Aquí Vázquez muestra que una de esas condiciones resulta de la necesidad de preparar su práctica a través de la individualización de qué información se pretende obtener de ella, qué tipo de conocimiento es relevante a esos efectos $-\mathrm{y}$ con ello qué expertos/as se requieren-y qué técnicas o métodos lo rigen. Otra condición resulta de la identificación de generalizaciones expertas adecuadamente soportada por información empírica. Para ello se requiere, al menos, inversión de recursos económicos para fomentar el desarrollo de la ciencia forense y tender puentes entre las comunidades expertas y las comunidades judiciales. 
de un plan institucional de esta envergadura; también es posible prever que su aplicación tomaría una perspectiva diferente en los casos concretos.

En cuanto a las herramientas procesales, Herdy afirma que ellas pueden incluso resultar compatibles con su modelo de deferencia crítica. Sin embargo, entiendo que bajo ese modelo estas herramientas, primero, serán relevantes en tanto fortalezcan las razones de esa deferencia, pero resultarán menos adecuadas si persiguen un fortalecimiento de la evaluación de la fiabilidad de la información en sí, que es justamente el objetivo de Vázquez. Además, sin mayores distinciones, admite tribunales integrados solo por expertos, algo que Vázquez sostiene como ultima ratio y sin desplazamiento de los/as jueces/zas. ${ }^{19}$

En diversos tramos, por su parte, Rimoldi visita y revisita las propuestas de Vázquez en el marco de las dos cuestiones epistemológicas que considera relevantes para la justificación racional de la inferencia probatoria pericial, esto es, su modelo de argumentación deferencial y los postulados del contextualismo epistémico que presenta.

Con base en ese modelo, examina cómo debería ser la gestión de información pericial en la admisión, práctica y valoración de la pericia. Coincide con Vázquez en que la individualización de líneas de trabajo a esos efectos puede hacer más eficiente el intercambio epistémico entre peritos/as y jueces/zas, a los que caracteriza como comunidad epistémica. ${ }^{20}$ Paralelamente, Vázquez y Rimoldi identifican ciertas decisiones institucionales que deben darse por fuera de los procesos particulares. Puntualmente, aunque en el marco de la constitución de la comunidad o la división de trabajo cognitivo respectivamente, ambas destacan la necesidad de invertir en ciencia forense que, siendo constitutivamente falible, requiere la evaluación constante de sus

19 Aquí observo cierta inconsistencia entre el modelo de la comprensión que presenta Vázquez y algunas de sus herramientas. Ello en la medida que, como se apunta notas más arriba, niega que información que no puede ser objeto de comprensión del/la juez/a ingrese al proceso; pero, a la par, admite como una herramienta procesal útil integrar el tribunal con peritos/as en casos muy complejos (por la razón que fuere) cuya información al juez o jueza no le sea asequible por otras herramientas.

${ }^{20}$ Vázquez en sus réplicas rechaza que la constitución de ese intercambio en términos de "comunidad" resulte adecuada según el modo en que funciona el mundo jurídico. En cambio, como se adelantó, estima que las relaciones entre los sujetos cognitivos —que a más de jueces/ zas y peritos/as, también ubica a las partes y a las comunidades expertas- son cooperativas. 
márgenes de error de sus conocimientos y métodos. Suplementariamente, y bajo su idea de comunidad, Rimoldi señala que su conformación favorece no solo la comprensión de los jueces como la identifica, sino también permite que los peritos disciernan las prácticas judiciales más relevantes, esto es, habilita que aprendan las partes del proceso, sus fines y consecuencias.

Por su parte, Rimoldi, como Herdy, sin excluir la argumentación comprensiva, revisan la utilización de indicadores indirectos en la evaluación de la inferencia probatoria pericial.

De modo central, Herdy apela al argumento de autoridad para articular su modelo de justificación por deferencia al perito, deferencia que resulta crítica - y por ende racional a su modo de ver- en la medida que permite al juez la revisión de los indicadores periféricos antes mencionados que configuran en su esquema razones de segundo orden. En una idea similar, pero en subsidio del elemento argumentativo, Rimoldi refiere que la exigencia de fiabilidad de la inferencia probatoria pericial exige relevar indicadores indirectos vinculados a criterios asociados al perito y al tipo de conocimiento. Las limitaciones que estas autoras reconocen - con diferente grado- a la autonomía de los jueces pueden reducirse a través de la consideración de esos criterios éticos o epistémicos.

Frente a estas ideas, Vázquez ofrece una nueva revisión sobre en qué medida deberían integrarse los criterios “indirectos" al análisis de la prueba pericial según sus ideas iniciales y las nuevas que presenta en sus réplicas. En lo central, niega que haya una distinción tajante entre criterios directos e indirectos, sino que ella es gradual y su uso depende de varios factores. Entre ellos, repara en la división del trabajo cognitivo expuesta en el contexto del caso concreto y de la etapa del proceso.

\section{Deudas pendientes en el análisis de la inferencia proba- toria pericial}

Mis comentarios en este apartado se dirigen a proponer ciertas deudas o caminos pendientes de recorrer en la discusión sobre la justificación de la inferencia probatoria pericial, tal como ha sido planteada. 
En primer lugar, se observa cierto grado de desconexión entre los argumentos y conclusiones analizados y las bases teóricas y normativas de la justificación del enunciado probatorio (punto 2). Un ideal de racionalidad al que aspiran los modelos de justificación de la inferencia probatoria pericial, y los diseños institucionales que cabe pensar a partir de ellos, debería poder mostrar cierto apoyo en las reglas - conceptuales, epistémicas y normativas- que rigen la justificación del razonamiento probatorio. Algo que hasta ahora parece menos explorado. Ello podría llevar - o no- a nuevas distinciones relevantes o reformular las utilizadas, o incluso tensar las bases mismas de la justificación racional de la decisión. En cualquier caso, creo que son reflexiones que deberían, al menos, intentarse con más énfasis.

En su faz dinámica, el razonamiento probatorio que justifica el enunciado probatorio, y que se integra a su vez por diversas inferencias probatorias como la pericial, adquiere diferentes dimensiones según los objetivos institucionales en juego, el momento procesal y quien lo formula. Las autoras han destacado muchos de estos factores que, como revelan, hacen a la complejidad de la justificación de esta inferencia probatoria. Pero, incluso bajo esas consideraciones, su fiabilidad al final dependerá del tipo valoración que se adopte y del estándar de prueba que se aplique a la decisión judicial. ${ }^{21}$

Más allá de la posición que se adopte en la materia, ${ }^{22}$ algunas simetrías o asimetrías deberían revisarse entre los estándares de prueba que rigen el enunciado probatorio y los elementos de juicio obtenidos a partir de la inferencia probatoria pericial. A modo de ejemplo, algunas de las ideas de las autoras se apoyan en nociones tales como creencia y aceptación de un enunciado probatorio, siendo que los vínculos entre ellas son elementales en el análisis de la suficiencia probatoria (Dei Vecchi, 2018, pp. 61-64); por lo que una expedición en ese sentido puede resultar provechosa, incluso para la identificación de los conceptos como comprensión o deferencia.

${ }^{21}$ Tampoco es menor en los tramos anteriores a la decisión — admisibilidad y práctica- qué enfoque -epistémico o no-debería primar a los efectos de pensar las razones que justifican o no cierto diseño institucional (conf. Bayón, 2009).

22 Solo por mencionar algunos trabajos que cubren el amplio espectro de problemas referidos a los estándares de prueba: Ferrer Beltrán, 2018; González Lagier, 2018; Dei Vecchi, 2020. 
Pericia, razonamiento probatorio y diseño institucional

En cuanto a la valoración de la prueba, la plausibilidad de todo o parte de los modelos de la comprensión, argumentación deferencial o de la deferencia crítica, estará condicionada a si ellos a su vez se apoyan en alguna versión atomista u holista referida al objeto y razones válidas para la justificación de la decisión judicial. ${ }^{23}$ Paralelamente, ello podría reconfigurar el análisis de cada elemento de la inferencia probatoria pericial, esto es, qué datos probatorios y generalizaciones expertas son relevantes y qué tipos de relaciones presentan entre sí.

Por su parte, y sin tomar partido en el problema de la demarcación entre qué es científico y qué no, también sería útil explorar algunas categorías respecto de las generalizaciones expertas, a fin de facilitar la tarea del juez o jueza. Es cierto, como apunta Vázquez, que los decisores y las decisoras muchas veces desconocen las condiciones que rigen la empresa científica o la expertise. Un estudio sistematizado de ellas podría evitar o reducir cierto prestigio, ganado con los años, de pruebas periciales basadas en conocimientos, métodos o técnicas poco fiables. ${ }^{24}$

Finalmente, me queda agradecer a los directores sucesivos de la revista por la invitación a editar y presentar esta sección, ${ }^{25}$ tarea que me ha enriquecido en el mientras tanto y sin dudas ha resultado muy gratificante al final. Va por ello también mi reconocimiento y gratitud a Carmen Vázquez por aceptar elaborar sus dos interesantes artículos. Y también a Florencia Rimoldi y Rachel Herdy por comprometerse especialmente con el debate de ideas. Ellas tres han conformado un valioso all-female panel.

${ }^{23}$ En ese sentido, seguir algunas tesis atomistas, podría condicionar la evaluación de la fiabilidad de los elementos de la inferencia pericial. Por ejemplo, los rangos de error expuestos en las generalizaciones expertas, o la identificación de los datos relevantes del caso, o sus relaciones no podrían ser contrastados con otros datos que permitan medir en el caso su fuerza probatoria. Del otro lado, si se sigue alguna versión holista, tal vez diseñar instituciones robustas en base a modelos de la comprensión disminuya su sentido, pues allí los esfuerzos argumentales finales responden a otros fines.

${ }^{24}$ Un ejemplo de este tipo de errores respecto de la prueba de huellas dactilares, ver Edmond, 2019.

${ }^{25}$ A ambos, a Hernán Bouvier con quien inició y a Federico Arena con quien finalizó. Igualmente me gustaría agradecer a Diego Dei Vecchi y Daniela Domeniconi por esas conversaciones itinerantes de las cuales tomé algunas ideas que rondan esta presentación, aunque los errores que puedan observarse son solo mi responsabilidad. 


\section{Bibliografía}

Accatino, D. (2014). Atomismo y holismo en la justificación probatoria. Isonomía, 40, 17-59.

Accatino, D. (2019). ¿Somos todos "racionalistas" ahora? Revus, 39, 85-102.

Anderson, T., Schum, D. y Twining, W. (2015). Análisis de la prueba. Madrid: Marcial Pons.

Bayón, J. C. (2008). Epistemología, moral y prueba de los hechos: hacia un enfoque no benthamiano. Analisi e Diritto, 15-34.

Bornstein, B. H. y Greene, E. (2017). The Jury Under Fire: Myth, Controversy, and Reform. New York: Oxford University Press.

Dei Vecchi, D. (2018). Problemas Probatorios perennes. Un análisis a la luz del nuevo proceso penal mexicano. México: Fontamara.

Dei Vecchi, D. (2020). La no tan sana crítica racional. En letra: Derecho Penal, 6(9), 40-55.

Edmond, G. (2019). Cuando el derecho es poco fiable: respuestas jurídicas a la prueba de huellas dactilares latentes. Quaestio Facti, 1, 301-355.

Ferrer Beltrán, J. (2005). Prueba y verdad en el derecho. Madrid-Barcelona, Marcial Pons.

Ferrer Beltrán, J. (2007). La valoración racional de la prueba. MadridBarcelona: Marcial Pons.

Ferrer Beltrán, J. (2018). Prolegómenos para una teoría sobre los estándares de prueba. El test case de la responsabilidad del Estado por prisión preventiva errónea. Ponencia presentada en el marco del Primer Congreso Mundial de Razonamiento Probatorio, Girona.

Gascón Abellán, M. (2004). Los hechos en el derecho. Bases argumentales de la prueba. Madrid-Barcelona: Marcial Pons.

González Lagier, D. (2018). ¿Es posible formular un estándar de prueba preciso y objetivo? Algunas dudas desde un enfoque argumentativo de la prueba. Ponencia presentada en el marco del Primer Congreso Mundial de Razonamiento Probatorio, Girona, 2018.

Marrero, D. (2015). Lineamientos generales para una epistemología jurídica. En A. Páez (coord.), Notas sobre hechos, evidencia y estándares de prueba. Ensayos de epistemología jurídica (pp. 15-36). Bogotá: Universidad de los Andes. 
Seidman, S. y Rose, M (2005). Real Juries. Annual Review of Law and Social Science, 1, 255-84.

Vázquez, C. (2015). De la prueba científica a la prueba pericial. MadridBarcelona: Marcial Pons.

Vázquez, C. (2018). La im/parcialidad pericial y otras cuestiones afines. Isonomía, 48, 69-107. 